\title{
Effect of the local hydrogen bonding network on the reorientational and translational dynamics in supercritical water
}

\author{
Ioannis Skarmoutsos ${ }^{a), b)}$ and Elvira Guardia ${ }^{a)}$ \\ Departament de Física i Enginyeria Nuclear, Universitat Politècnica de Catalunya, B4-B5 Campus Nord \\ UPC, 08034 Barcelona, Catalonia, Spain
}

(Received 26 October 2009; accepted 12 January 2010; published online 16 February 2010)

\begin{abstract}
Molecular dynamics simulations have been performed in a wide range of densities along a near critical isotherm of supercritical water in order to reveal the interconnection between the local hydrogen bonding (HB) network and several related dynamic properties. The results obtained have revealed a significant slowing down of reorientational dynamics of the water molecules as the value of the number of hydrogen bond per molecule increases and this is reflected on the increase in the reorientational correlation times. The calculated reorientational times exhibit also an increasing trend by increasing the bulk density, and this effect is more pronounced in the case of the first-order Legendre reorientational correlation functions. A clear nonlinear dependence of the librational mode frequencies of the water molecules on the augmented local density around them has also been revealed. This result could be regarded as an additional support of experimental observations suggesting the use of a nonlinear relation when analyzing the density dependence of spectroscopic peak frequencies in order to extract information about local density augmentation in supercritical fluids. The HB dynamics have been also investigated, revealing a plateau in the calculated HB lifetimes at intermediate and higher liquidlike densities and a small increase at low, gaslike densities. (c) 2010 American Institute of Physics. [doi:10.1063/1.3305326]
\end{abstract}

\section{INTRODUCTION}

Supercritical water (SCW) is one of the solvents that have attracted a significant attention of the scientific and engineering community due to its ability to serve as a medium in a wide range of chemical processes. ${ }^{1-4} \mathrm{SCW}$ is an environmental benign solvent and a cheap alternative to organic ones and has been used as a reaction media in several processes. The main reason for this wide applicability is that by slightly adjusting the thermodynamic conditions the miscibility of polar and nonpolar compounds in SCW could be significantly affected due to the changes in the dielectric properties of SCW, allowing thus the eclectic dissolution of several types of solutes in SCW. Moreover, the reaction kinetics in SCW could be also appropriately tuned and taking also into account that the viscosity of SCW remains relatively low even at liquidlike densities, mass transfer and diffusion-controlled reactions are enhanced in SCW. For all these reasons SCW has been used as a solvent, catalyst, or even reactant in numerous chemical and engineering processes such as chemical and material synthesis, hazardous waste treatment (oxidation of organic wastes), biomass processing, plastic recycling, production of combustion flames, etc. Additionally SCW is a solvent of great importance for geochemical processes, where it acts as a medium in several hydrothermal reactions in Earth's mantle. ${ }^{1}$

The properties of SCW exhibit, in general, significant

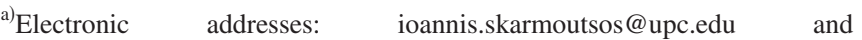
elvira.guardia@upc.edu.

${ }^{b)}$ Present address: Department of Chemistry, Imperial College London, London SW7 2AZ, United Kingdom.
}

differences with liquid state properties as these have been pointed out in the existing literature. ${ }^{5-18}$ However, previously reported experimental studies ${ }^{5-16}$ have revealed that a local hydrogen bonding (HB) network, which is the main characteristic of liquid water, still exists in the supercritical state, even though it is significantly weaker. Moreover, previously reported simulation studies ${ }^{17-32}$ have also confirmed the existence of this HB network by investigating the pressure and temperature effect on the creation of hydrogen bonds between the water molecules. One other characteristic property of SCW is the existence of significant local density augmentation effects. One of the authors revealed in quite recent publications $\mathrm{s}^{24,33}$ that the local density augmentation and enhancement factors are much more pronounced in the case of $\mathrm{SCW}$ in comparison with other $\mathrm{HB}$ and non-HB fluids. ${ }^{24,34-36}$ This behavior has been related to the nonlinear density dependence of the mean number of hydrogen bonds per water molecule, which exhibits much greater deviations form linearity in comparison with other HB fluids such as alcohols and ammonia. ${ }^{24,34,36,37}$ Taking into account all these previous findings, a systematic investigation of the possible interconnection between the HB structure and dynamics, local density inhomogeneities, and related single and collective dynamic properties could provide a deeper insight about fundamental issues on the behavior of SCW.

The present study has been devoted to the thorough investigation of all the aforementioned issues by performing a series of molecular dynamics (MD) simulations of SCW in a near critical isotherm. Such a treatment could provide important information on many open questions regarding these issues and is the main aim of this work. This work continues 
TABLE I. Summary of the simulated thermodynamic state points for supercritical water.

\begin{tabular}{lc}
\hline \hline & $\mathrm{T}=666 \mathrm{~K}^{\mathrm{a}}$ \\
\hline Simulated state points & $\begin{array}{c}\rho^{\mathrm{a}} \\
\left(\mathrm{g} / \mathrm{cm}^{3}\right)\end{array}$ \\
\hline A & 0.061 \\
B & 0.114 \\
C & 0.196 \\
D & 0.277 \\
E & 0.368 \\
F & 0.408 \\
G & 0.461 \\
H & 0.504 \\
I & 0.644 \\
\hline \hline
\end{tabular}

${ }^{\mathrm{a}}$ The critical parameters for water are $\mathrm{T}_{c}=647.1 \mathrm{~K}$ and $\rho_{c}=0.322 \mathrm{~g} / \mathrm{cm}^{3}$.

our systematic investigations on the local density inhomogeneities and corresponding dynamics in supercritical fluids, as well as our previous studies on the effect of the local HB network on single reorientational dynamics in supercritical ethanol. $^{37}$ This paper is organized as follows: The computational details of the performed simulations are presented in Sec. II. The results obtained and the following discussion upon them are presented in Sec. III. Finally, Sec. IV contains the general conclusions and remarks drawn from the present study.

\section{SIMULATION DETAILS}

Several MD atomistic simulations of SCW were performed at a constant temperature of $666 \mathrm{~K}$ and for a series of densities in the range of $0.2-2.0 \rho_{c}$. The simulated state points of SCW are presented in Table I. The simulations were carried out with 500 molecules, using the MOSCITO 4.140 software. $^{38}$ Each simulation was extended to $500 \mathrm{ps}$ to achieve equilibrium, starting from an initial face centered cubic configuration, and the properties of SCW were evaluated in subsequent simulations with duration 500 ps. In all simulations the equations of motion were integrated using a leapfrog-type Verlet algorithm and the integration time step was set to 1 fs. The Berendsen thermostat ${ }^{39}$ with a temperature relaxation time of $0.5 \mathrm{ps}$ was also used to constrain the temperature during the simulations. The intramolecular geometry of the molecules was also constrained by employing the shake algorithm. ${ }^{40}$

The rigid simple point charge/extended (SPC/E) potential model ${ }^{41}$ was employed to describe the site-site interactions between the water molecules. This three-site potential model has been successfully used in previous studies of liquid and supercritical water, as well as in the case of several aqueous mixtures. Therefore, our choice has been based on previously reported simulation results concerning the accuracy of this model in reproducing reasonably various properties of the fluid. ${ }^{4-45}$ In our simulations a cutoff radius of 12.0 $\AA$ has been applied for Lennard-Jones interactions and long-range corrections have been also taken into account. Moreover, to account for the long-range electrostatic interac- tions the Ewald summation technique was used based on the more exact approximation of the Newton-Gregory forward difference interpolation scheme. ${ }^{38}$

\section{RESULTS AND DISCUSSION}

\section{A. HB and single reorientational dynamics}

As mentioned in Sec. I, one of the authors in a previous publication $^{24}$ performed a static HB analysis of SCW and found that the mean number of hydrogen bonds per water molecule exhibits a clearly nonlinear density dependence, which is much more pronounced in SCW in comparison with other HB supercritical fluids such as methanol, ${ }^{24}$ ethanol, ${ }^{37}$ and ammonia. ${ }^{24}$ This behavior has been related as one of the main reasons for the observation of strong local density augmentation effects in SCW. The HB statistics have also revealed that this nonlinear density dependence is mainly a result of the nonlinear density dependence of the fraction of water molecules forming one and two hydrogen bonds, whereas in the case of the fraction of molecules forming three bonds the density dependence is linear.

In the present treatment we decided to investigate further the density dependence of the corresponding HB lifetimes, as well as to investigate the effects of the HB state of the water molecules on their corresponding reorientational dynamics. One of the main motivations for this study was a previous study that we had performed for supercritical ethanol. ${ }^{37}$ In that study we had revealed that the reorientational dynamics of the individual ethanol molecules were very sensitive on the HB state of the molecules and the dynamics of HB-free molecules were significantly different than those corresponding to molecules forming one or two hydrogen bonds. Therefore we decided to investigate these effects for SCW, which is the most characteristic HB fluid, in order to reveal more information on the interconnection of the local HB network in supercritical fluids with single molecule dynamics.

According to literature, the average $\mathrm{HB}$ dynamics for pairs $i, j$ of hydrogen bonded molecules could be described using the following time correlation function (tcf): ${ }^{46-52}$

$$
C_{\mathrm{HB}}(t)=\frac{\left\langle h_{i j}(0) h_{i j}(t)\right\rangle_{t^{*}}}{\left\langle h_{i j}(0)^{2}\right\rangle} .
$$

The corresponding HB lifetime is defined as

$$
\tau_{\mathrm{HB}}=\int_{0}^{\infty} C_{\mathrm{HB}}(t) d t .
$$

The variable $h_{i j}$ has been defined in the following way:

$h_{i j}(t)=1$

if molecule $j$ is hydrogen bonded with molecule $i$ at

times 0 and $t$ and the bond has not been broken in the

meantime for a period longer than $t *$,

$h_{i j}(t)=0 \quad$ otherwise.

Of course, using this definition, the calculation of $C_{\mathrm{HB}}(t)$ depends on the selection of the parameter $t^{*}$. The two limiting cases arising from this definition are as follows. 

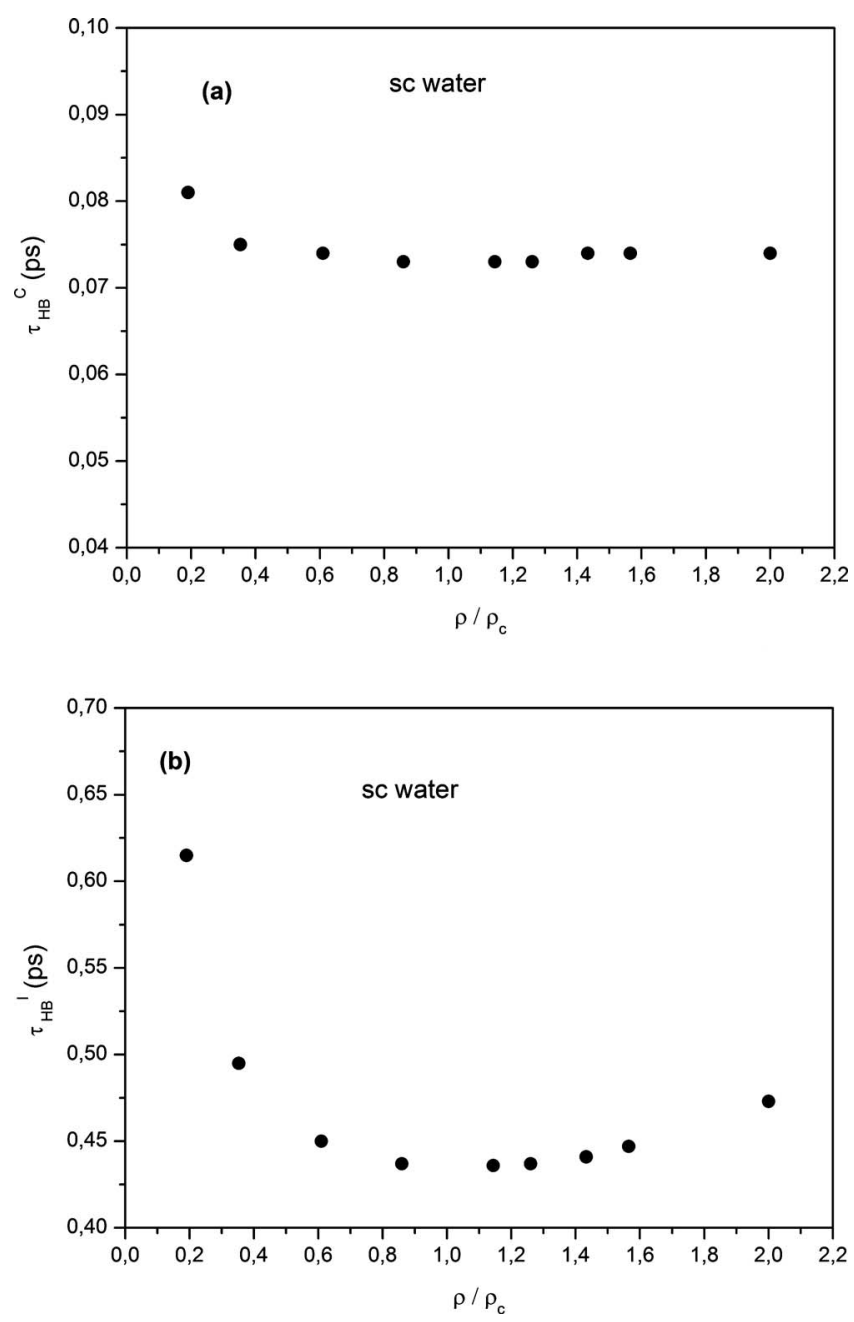

FIG. 1. Density dependence of the calculated: (a) continuous, $\tau_{\mathrm{HB}}{ }^{C}$, and (b) intermittent, $\tau_{\mathrm{HB}}^{I}$, HB lifetimes of SCW.

(a) If $t^{*}=0$, which represents the so-called continuous definition. In this case the calculated tcf is the continuous one $C_{\mathrm{HB}}{ }^{C}(t)$ and the corresponding lifetime is the continuous lifetime $\tau_{\mathrm{HB}}{ }^{C}$.

(b) If $t^{*}=\infty$, which represents the so-called intermittent definition. In this case the calculated tcf is the intermittent one $C_{\mathrm{HB}}{ }^{I}(t)$ and the corresponding lifetime is the intermittent lifetime (or HB relaxation time) $\tau_{\mathrm{HB}}{ }^{I}$.

These two definitions describe very different aspects of HB dynamics and have been well described in our previous publications. In the case of SCW, in order to define whether a pair of water molecules is hydrogen bonded, we employed a commonly used geometric criterion, ${ }^{21,53}$ used also in the previously mentioned ${ }^{24}$ static HB analysis of SCW. According to this criterion a hydrogen bond between two water molecules exists if the interatomic distances are such that $R_{\mathrm{O} \ldots \mathrm{O}} \leq 0.36 \mathrm{~nm}, R_{\mathrm{H} \ldots \mathrm{O}} \leq 0.24 \mathrm{~nm}$ and the donor-acceptor angle $\mathrm{H}-\mathrm{O} \ldots \mathrm{O} \leq 30^{\circ}$ (the symbol...corresponds to intermolecular distances).

The density dependence of the calculated continuous, $\tau_{\mathrm{HB}}{ }^{C}$, and intermittent, $\tau_{\mathrm{HB}}{ }^{I}, \mathrm{HB}$ lifetimes of SCW is presented in Fig. 1. From this figure we may see that at low densities the values of the HB lifetimes are higher, especially in the intermittent case, and then we may observe a decrease in these lifetimes, which exhibit a plateau at intermediate and higher densities. This finding comes in agreement with the previous assumptions, according to which at low densities the molecules form small metastable clusters with larger lifetimes. ${ }^{24,36,37}$ This behavior has been also related to the corresponding slower local density reorganization at the low density region. The density dependence of these HB lifetimes is also similar to the one obtained for residence times corresponding to the first shell of $\mathrm{SCW},{ }^{42}$ which is located in very short intermolecular distances, in a previous publication of one of the authors. This finding indicates that the behavior of pair dynamic properties at short intermolecular distances, such as the residence and HB dynamics, is similar.

In order to investigate the effect of the HB state of the water molecules on their reorientational dynamics we calculated the Legendre reorientational tcfs as a function of the hydrogen bonds per molecule. These functions can be defined as follows: ${ }^{37}$

$$
\begin{aligned}
C_{n, \ell R}(t)= & \frac{\left\langle P_{\ell}(\vec{u}(0) \cdot \vec{u}(t)) \cdot \Theta_{n}(0)\right\rangle}{\left\langle\Theta_{n}(0)\right\rangle}, \\
& n=0,1,2,3 \text { and } \ell=1,2 .
\end{aligned}
$$

In this equation $\vec{u}$ is a unit vector along a specified direction inside a molecule and $P_{\ell}$ is a Legendre polynomial $\left[P_{1}(x)\right.$ $\left.=x, P_{2}(x)=1 / 2\left(3 x^{2}-1\right)\right]$. The index $\ell$ defines the order of the Legendre polynomial, whereas the index $n$ defines the instantaneous number of hydrogen bonds which forms a molecule at each time $t$.

The function $\Theta_{n}(t)$ is defined as follows:

$\Theta_{n}(t)=1$, if a molecule forms $n$ hydrogen

bonds at time $t$,

$\Theta_{n}(t)=0, \quad$ otherwise

The corresponding reorientational times are defined as

$$
\tau_{n, \ell R}=\int_{0}^{\infty} C_{n, \ell R}(t) d t, \quad n=0,1,2,3 \quad \text { and } \quad \ell=1,2
$$

We have to mention that a similar definition has been employed in a recent study, ${ }^{54}$ where the effect of the solvation number on the translational and rotational dynamics of SCW was investigated. In this study we investigated the reorientational dynamics of molecules forming $n=0$ (HB-free molecules), $n=1, n=2$, and $n=3$ hydrogen bonds, in order to see the effect of the local HB network on these properties. This analysis was performed for both the $\mathrm{O}-\mathrm{H}$ intramolecular bond vectors, whose dynamics is closely related to the hydrogen bonds formed among the water molecules. At this point we have to mention also that the average $\mathrm{O}-\mathrm{H}$ secondorder reorientational times calculated with the SPC/E potential model have been found to be in very good agreement with NMR experimental data, as it has been revealed in a previous study of one of the authors. ${ }^{55}$ The calculated first and second-order Legendre tcfs for some representative state points are depicted in Figs. 2 and 3 and the density dependence of the corresponding reorientational times is presented in Figure 4. The effect of the HB state on the obtained reori- 

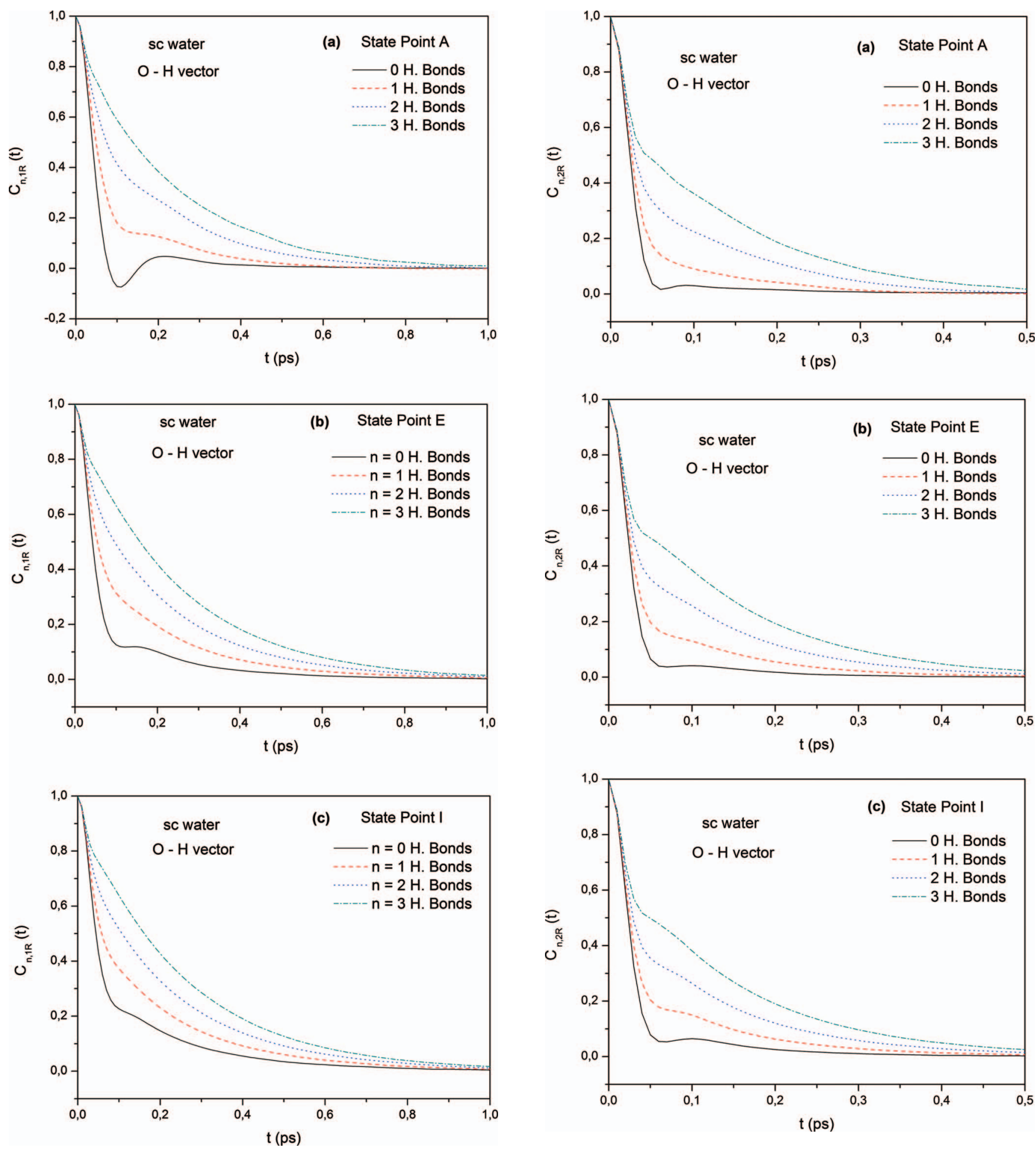

FIG. 2. The calculated first-order Legendre reorientational tcfs, $C_{n, 1 R}(t)[\mathrm{Eq}$. (4)] for some representative state points.

entational times is also depicted for several representative simulated state points in Fig. 5 .

From the results obtained we may clearly see that the HB state of the water molecules affects their reorientational dynamics. We may observe that especially the reorientational dynamics of HB-free molecules $(n=0)$ are substantially different and their corresponding correlation times are lower in comparison with those obtained for hydrogen bonded molecules. Especially at low density thermodynamic state points

FIG. 3. The calculated second-order Legendre reorientational tcfs, $C_{n, 2 R}(t)$ [Eq. (4)] for some representative state points.

[see Fig. 2(a)], we may observe that the first-order Legendre reorientational function $C_{0,1 R}(t)$ has a negative part at a quite short time scale and resembles a gas reorientational motion. However, as the values of $n$ increase, this negative part disappears and we may observe a more liquidlike behavior. The physical meaning of this observation is that as the value of the hydrogen bonds per water molecule increases, the local HB network around each individual water molecule starts to become more cohesive, resembling a liquidlike environment, 

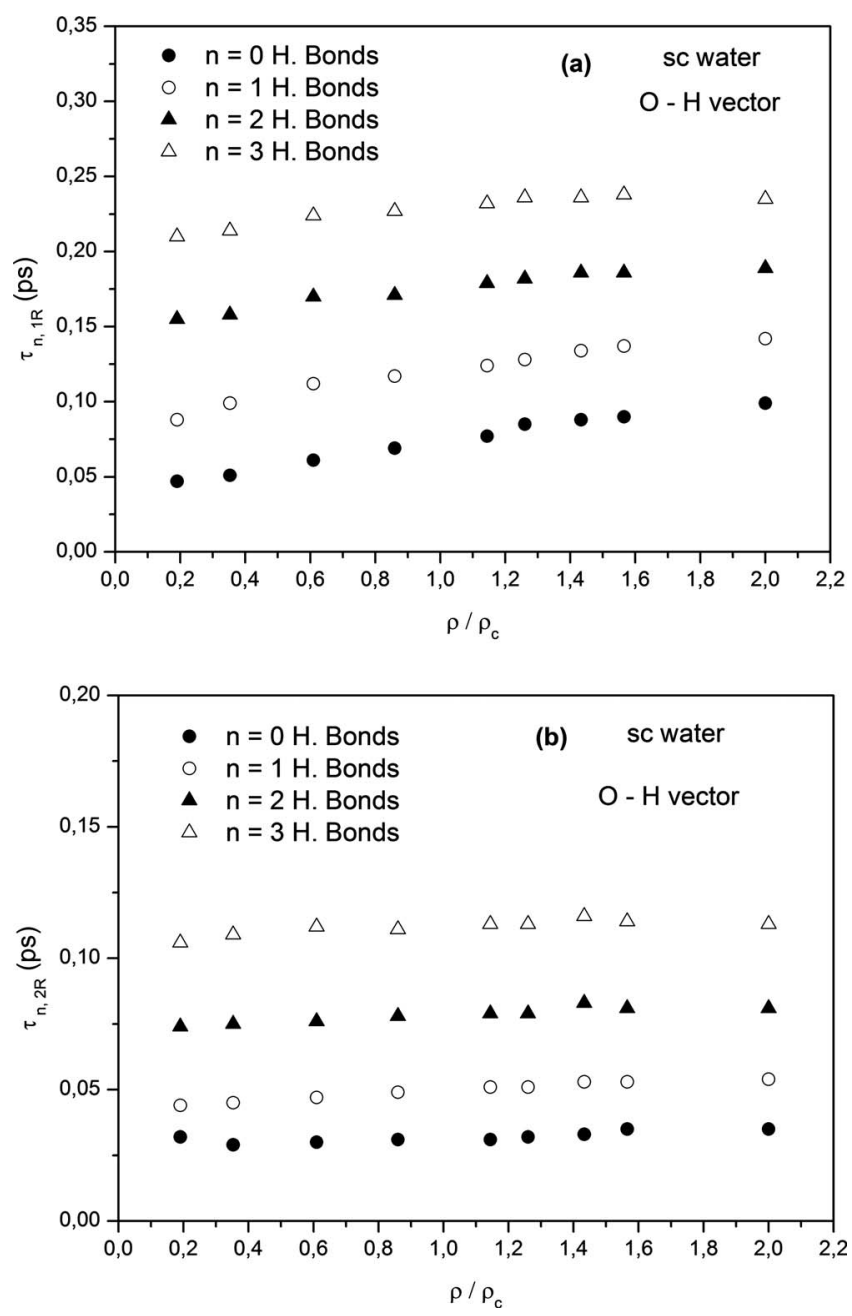

FIG. 4. Density dependence of the calculated: (a) first, $\tau_{n, 1 R}$, and (b) second, $\tau_{n, 2 R}$ order Legendre reorientational correlation times.

and this change in the local environment affects strongly the reorientational motion of the water molecules. In general, we may observe a slower decay in the reorientational tcfs $C_{n, 1 R}(t)$ as the values of $n$ increase and this slowing down is reflected on an increase in the calculated reorientational correlation times $\tau_{n, \ell R}$. If the bulk density is also increased, then the local HB network around each water molecule becomes even more cohesive and this results in a significant retardation on the decay of the reorientational correlation functions. Moreover, we have revealed that this behavior is more pronounced in the case of the first-order Legendre reorientational dynamics, whereas in the case of the second-order Legendre reorientational dynamics and especially for HBfree molecules, the corresponding reorientational times are not significantly affected by the bulk density. The sensitivity of the second-order reorientational times on the local HB network has also been mentioned in previous studies, ${ }^{56}$ where it was also discussed that the effect of the local HB network is stronger in the liquid state than at supercritical conditions. Therefore, we may say that the first-order Legendre reorientational times could be considered as a better probe to analyze the effect of the local HB network on the reorientational dynamics in SCW.

This result comes in agreement with our previous find-
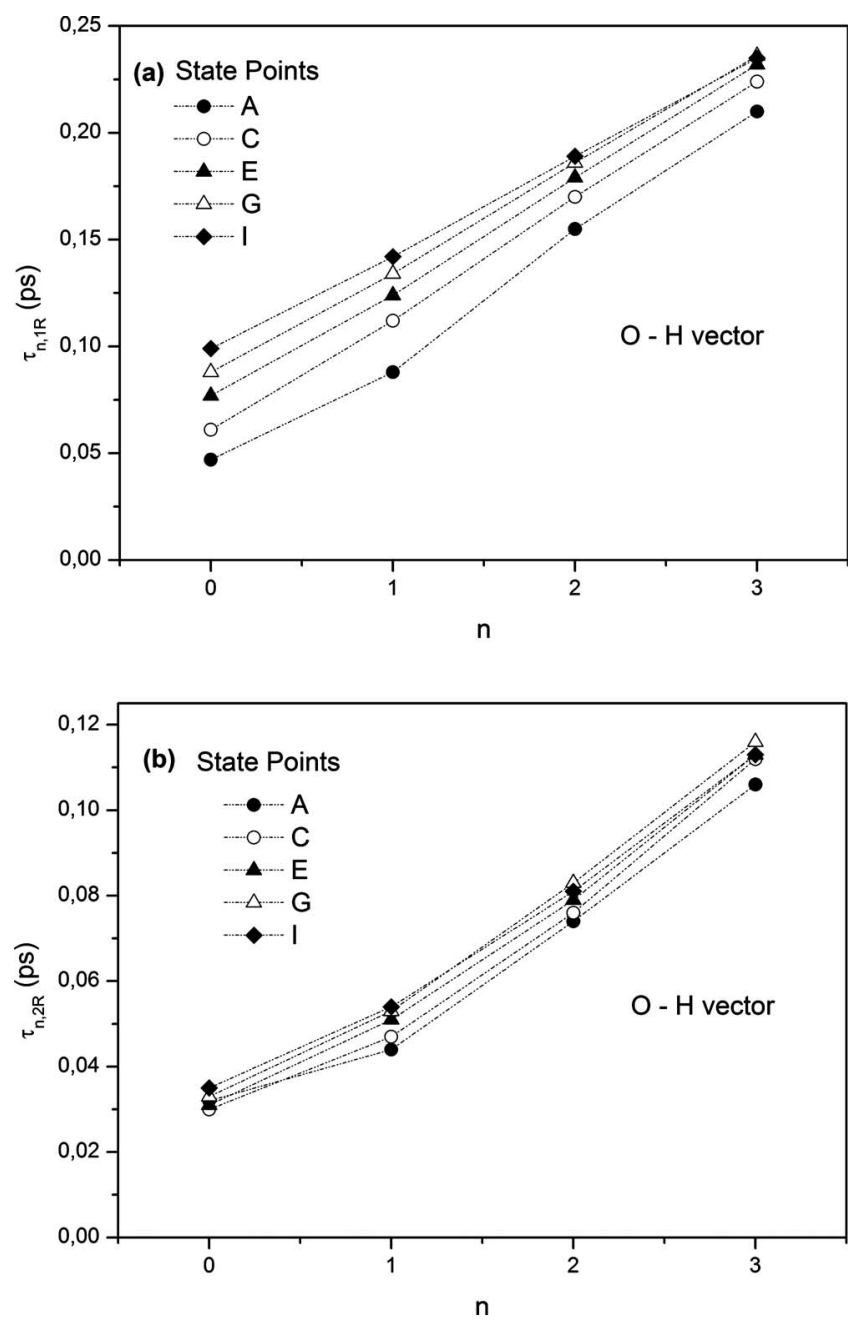

FIG. 5. (a) First, $\tau_{n, 1 R}$, and (b) second, $\tau_{n, 2 R}$, order Legendre reorientational correlation times as a function of the number of $\mathrm{H}$. Bonds formed by a water molecule for several representative state points.

ings in the case of ethanol, ${ }^{37}$ where we have revealed that in the cases where the interactions among the molecules are relatively weak, the second-order Legendre reorientational dynamics are not significantly affected by the bulk density of the system in contrast with the behavior of the first-order ones, which are more strongly affected. This fact indicates that these two different ways of depicting and analyzing molecular reorientation in fluids are differently influenced by the local environment. However, when these interactions become stronger, like in the case of more strongly hydrogen bonded molecules, the local HB network affects more significantly the reorientational dynamics of the molecules and this effect is clearly reflected on the density dependence of the calculated first and second-order reorientational times.

\section{B. Translational, librational dynamics}

In the present treatment we have also focused on the effect of the local structural network of the water molecules on the single translational dynamics of SCW. To do so, we have calculated the appropriate atomic velocity tcfs, as well as the center of mass (CM) velocity tcfs for all the simulated 
state points. In the case of the hydrogen atomic velocities the average velocity tcfs are have been calculated using the relation

$$
C_{v}{ }^{\mathrm{H}}(t)=\frac{\left\langle\vec{v}_{\mathrm{H}}(0) \cdot \vec{v}_{\mathrm{H}}(t)\right\rangle}{\left\langle\vec{v}_{\mathrm{H}}(0)^{2}\right\rangle} .
$$

The corresponding spectral densities $S_{v}{ }^{\mathrm{H}}(\omega)$ have been also calculated by performing a Fourier transform on the $C_{v}{ }^{\mathrm{H}}(t)$ tcfs,

$$
S_{v}{ }^{\mathrm{H}}(\omega)=\int_{0}^{\infty} \cos (\omega t) C_{v}^{\mathrm{H}}(t) d t .
$$

The $S_{v}{ }^{\mathrm{H}}(\omega)$ have been calculated by numerical integration using a Bode rule, after applying a Hanning window to the calculated atomic velocity tcfs. Our main motivation to calculate these spectral densities was to estimate the density dependence of the libration peak frequencies in SCW and to relate them with the local environment around a water molecule. Although several interesting studies have been already presented in literature devoted to the translational and librational dynamics of water molecules in the supercritical state ${ }^{54,57-59}$ we have to note that this is the first attempt to directly relate these dynamics with the local density augmentation effects in SCW. According to literature, ${ }^{24,60-70}$ the experimental determination of local density augmentation effects in supercritical fluids is usually detected indirectly by measuring spectroscopically some molecule-centered observable quantity that is sensitive to the fluid's local density around it. One of our motivations to perform this analysis was a previous debate in literature concerning the experimental observation of the local density augmentation in supercritical fluoroform. Saitow et al. ${ }^{66-69}$ performed spectroscopic measurements of pure supercritical fluoroform and methanol. According to their experiments, the local density augmentation in methanol is more pronounced than in fluoroform. However, the results obtained by previous MD simulations of supercritical methanol ${ }^{34}$ and fluoroform ${ }^{62}$ have estimated sufficiently smaller local density augmentation values for both fluids. Song and Maroncelli ${ }^{62}$ systematically examined the disagreement between their simulation and experiment in the case of supercritical fluoroform. In that paper Song and Maroncelli pointed out that a possible reason for this discrepancy could be the assumption of a linear proportionality between spectral shifts and local densities as well as the methodology employed to derive local densities from the Raman shifts observed by Saitow et al. ${ }^{67}$

In general, several recent studies have provided information about the density dependence of spectroscopic observables ${ }^{24,33,34,62,71-73}$ in supercritical fluids. From these studies we may see that, in general, there is a nonlinear bulk density dependence; however the relation between these spectroscopic observables with the local density is still not very clear. In some papers Maroncelli et al. ${ }^{63}$ also tried to propose some models in order to relate experimental spectral shifts with the refractive index and the dielectric constant, using empirical nonlinear relations for the dependence of the refractive index and the dielectric constant on the local density. In other cases the authors modeled separately attractive and repulsive contributions to the spectral shifts, assuming linear bulk density dependence in the case of attractive contributions and a perturbed hard sphere model in the case of repulsive contributions. ${ }^{66,69,70}$ On the other hand, in the case of MD simulations it is possible to directly determine in the same time the local density around the molecules as well as a spectroscopic observable, and see if they exhibit a linear relation or not. Therefore, the use of MD simulations could be used as very helpful tool in order to have a more straightforward determination of the relation between vibrational frequencies and local densities.

In this study we tried to directly relate some spectroscopic observable quantities with the average effective local density, $\rho_{\text {eff }, l}$, around the water molecules. The use of effective local densities to investigate local density inhomogeneities in supercritical fluids has been extensively discussed and justified in previous publications. ${ }^{33,34,61,62}$ To calculate the effective local densities in SCW, we initially calculated the average coordination number $\mathrm{N}_{c}$ corresponding to the first solvation shell of SCW at each state point,

$$
\mathrm{N}_{c}\left(\rho, R_{c}\right)=4 \pi \rho \int_{0}^{R_{c}} g_{\text {com }}(r) r^{2} d r
$$

In this equation, $g_{\text {com }}(r)$ is the $\mathrm{CM}$ pair radial distribution function and $\rho$ is the bulk number density of the fluid. $R_{c}$ is a defined cutoff distance specifying the first solvation shell around a water molecule. To estimate the excess local density in a supercritical fluid, it is more convenient to calculate the effective local density, ${ }^{33,34,61,62} \rho_{\text {eff }, l}$, according to the following relation:

$$
\rho_{\mathrm{eff}, l}\left(\rho, R_{c}\right)=\frac{\mathrm{N}_{c}\left(\rho, R_{c}\right)}{\mathrm{N}_{c}\left(\rho_{\text {ref }}, R_{c}\right)} \rho_{\text {ref }} .
$$

The employed reference density, $\rho_{\text {ref }}$, corresponds typically to a high liquidlike density and has been set as $2 \rho_{c}$. The cutoff distance $R_{c}$ is determined as the position of the first minimum of the corresponding $g_{\text {com }}(r)$ observed at this high reference density $\rho_{\text {ref }}$. In the case of SCW the calculated value of $R_{c}$ is $4.22 \AA$. This cutoff distance has been used to compute the effective local density, $\rho_{\text {eff }, l}$, of the first coordination shell in all the simulated state points of interest.

By calculating the spectral densities $S_{v}{ }^{\mathrm{H}}(\omega)$, we estimated the libration peak frequencies of SCW, $\omega_{v \text {, max }} \mathrm{H}$, which are located in the far infrared region. Afterward, we investigated the relation of $\omega_{v, \text { max }} \mathrm{H}$ with the calculated effective local density $\rho_{\text {eff }, l}$. The calculated spectral densities $S_{v}{ }^{\mathrm{H}}(\omega)$, as well as the dependence of the libration peak frequencies $\omega_{v, \max }^{\mathrm{H}}$ on $\rho_{\text {eff, }, l}$ are presented in Fig. 6. From this figure we may clearly see that this relation is clearly nonlinear, supporting thus the recently published hypotheses. We have to mention here that we presented this analysis in the case of the hydrogen atomic velocities since in the case of the oxygen ones, the corresponding spectral densities do not exhibit these bands, in contrast to their behavior at the liquid state. ${ }^{22}$

In order to investigate the effect of the HB state of the water molecules on the translation dynamics, we calculated the appropriate velocity tcfs, defined as follows: 

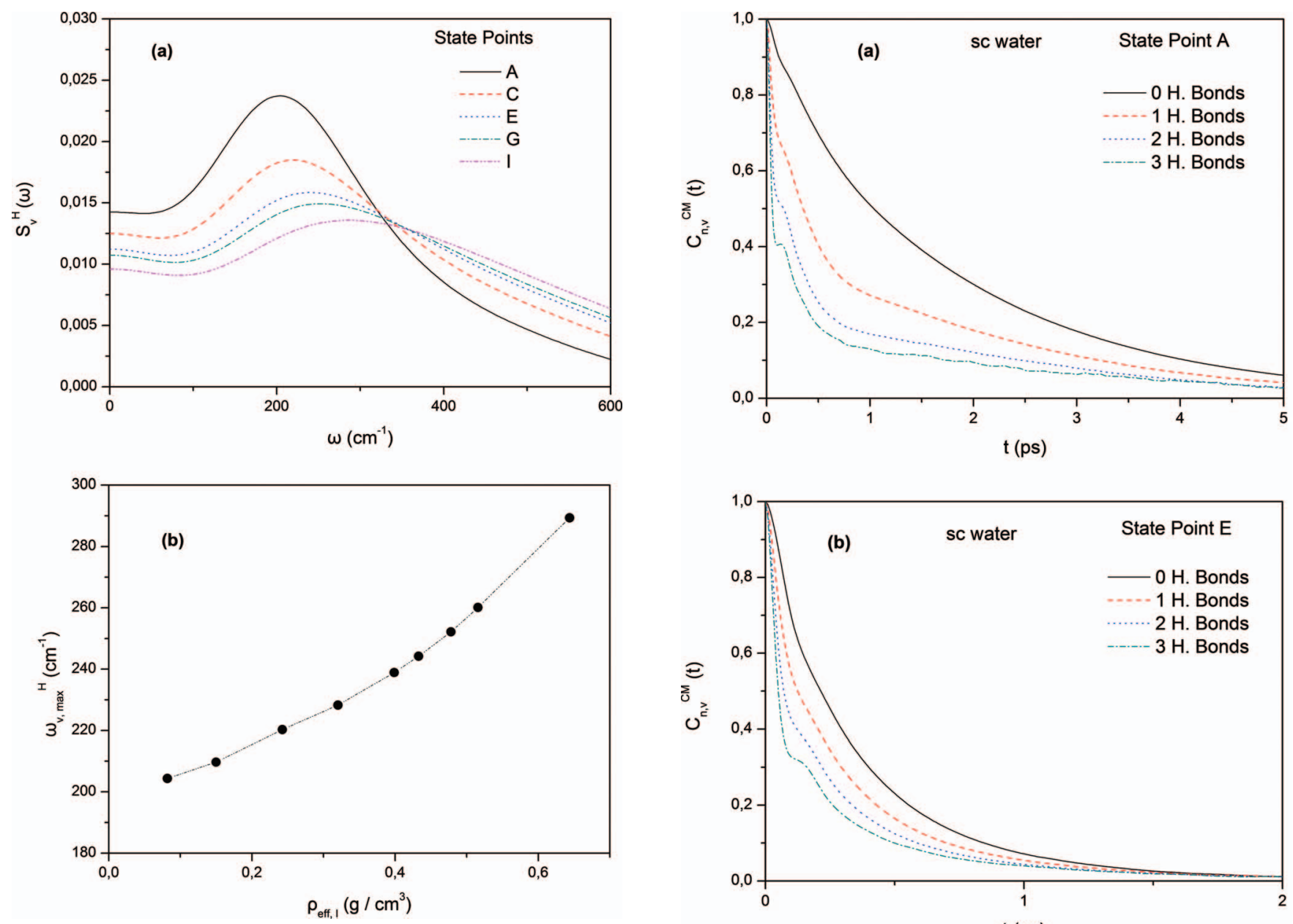

FIG. 6. (a) The calculated average spectral densities $S_{v}{ }^{\mathrm{H}}(\omega)$. (b) Dependence of the average libration peak frequencies $\omega_{v \text {,max }}{ }^{H}$ on the average effective local densities of SCW, $\rho_{\text {eff }, l}$.

$$
\begin{aligned}
C_{n, v}{ }^{i}(t)= & \frac{\left\langle\vec{v}_{i}(0) \cdot \vec{v}_{i}(t) \cdot \Theta_{n}(0)\right\rangle}{\left\langle\vec{v}_{i}(0)^{2} \cdot \Theta_{n}(0)\right\rangle}, \\
& i \equiv \mathrm{H}, \mathrm{CM}, \quad n=0,1,2,3 .
\end{aligned}
$$

The corresponding velocity correlation times are defined as

$$
\tau_{n, v}{ }^{i}=\int_{0}^{\infty} C_{n, v}{ }^{i}(t) d t, \quad i \equiv \mathrm{H}, \mathrm{CM}, \quad n=0,1,2,3 .
$$

In the case of the hydrogen atomic velocity tcfs we also calculated the corresponding spectral densities,

$$
S_{n, v}{ }^{\mathrm{H}}(\omega)=\int_{0}^{\infty} \cos (\omega t) C_{n, v}{ }^{\mathrm{H}}(t) d t, \quad n=0,1,2,3 .
$$

The results obtained are presented in Figs. 7-9. From these figures, we may clearly observe that the translational dynamics are also very sensitive on the HB state of the water molecules and, in general, we may see a nonlinear decrease in the correlation times $\tau_{n, v}{ }^{\mathrm{CM}}$ with density. Additionally, we also depicted the dependence of $\tau_{n, v}{ }^{\mathrm{CM}}$ on the number of hydrogen bonds $n$ formed by a molecule for several representative state points. From Fig. 8 we may see that, in general, $\tau_{n, v}{ }^{\mathrm{CM}}$ decreases when $n$ increases and while at lower densities, exhibits a nonlinear behavior; at higher and more
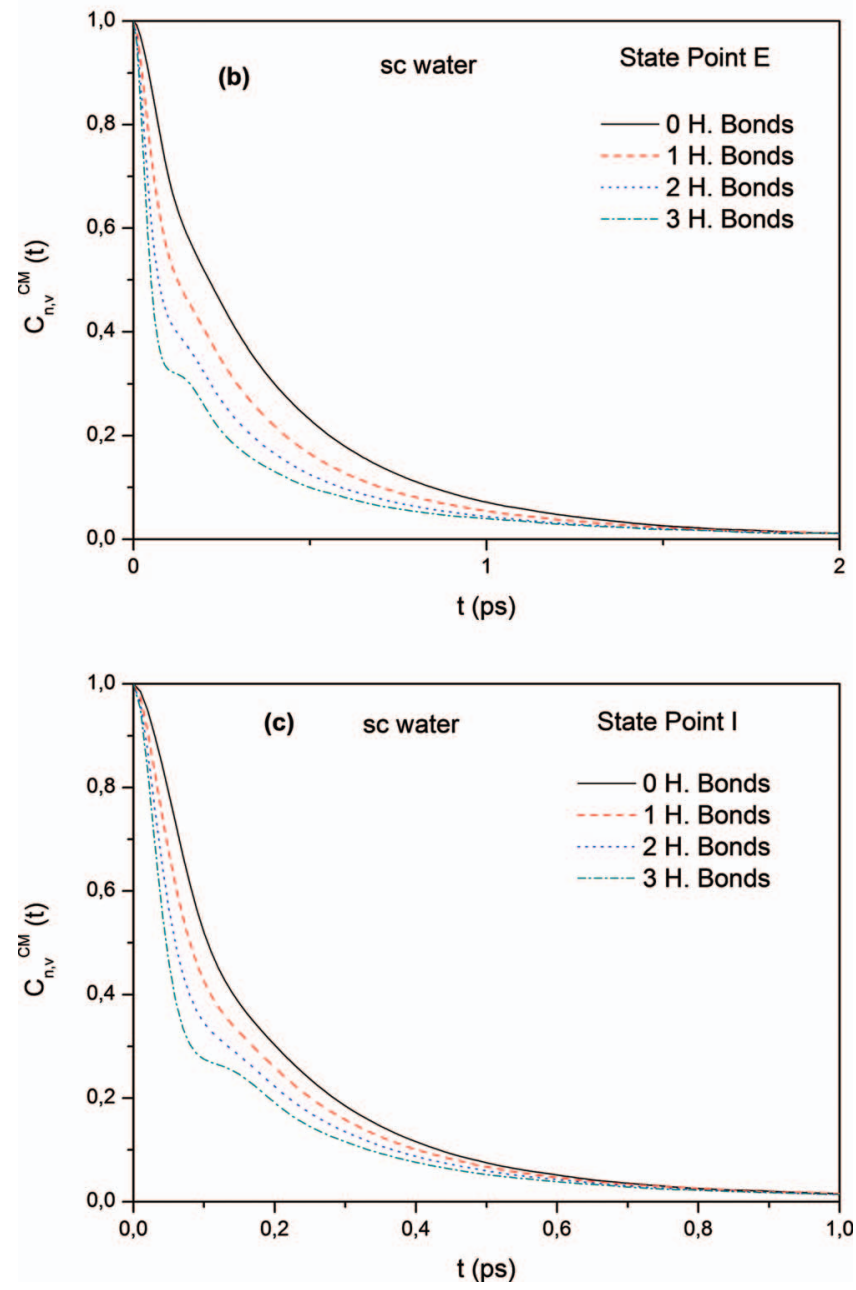

FIG. 7. The calculated CM velocity tcfs $C_{n, v}{ }^{\mathrm{CM}}(t)$ [Eq. (9)] for some representative state points.

similar to liquidlike densities this behavior is clearly linear. Concerning the libration peak frequencies corresponding to the calculated spectral densities $S_{n, v}{ }^{\mathrm{H}}(\omega)$ we may observe from Fig. 9 that as the number of hydrogen bonds $n$ increases the corresponding peaks of $S_{n, v}{ }^{\mathrm{H}}(\omega)$ are also shifted toward higher frequencies for all the investigated state points. This finding clearly indicates that the libration frequency of the water molecules is directly related to the local HB network 

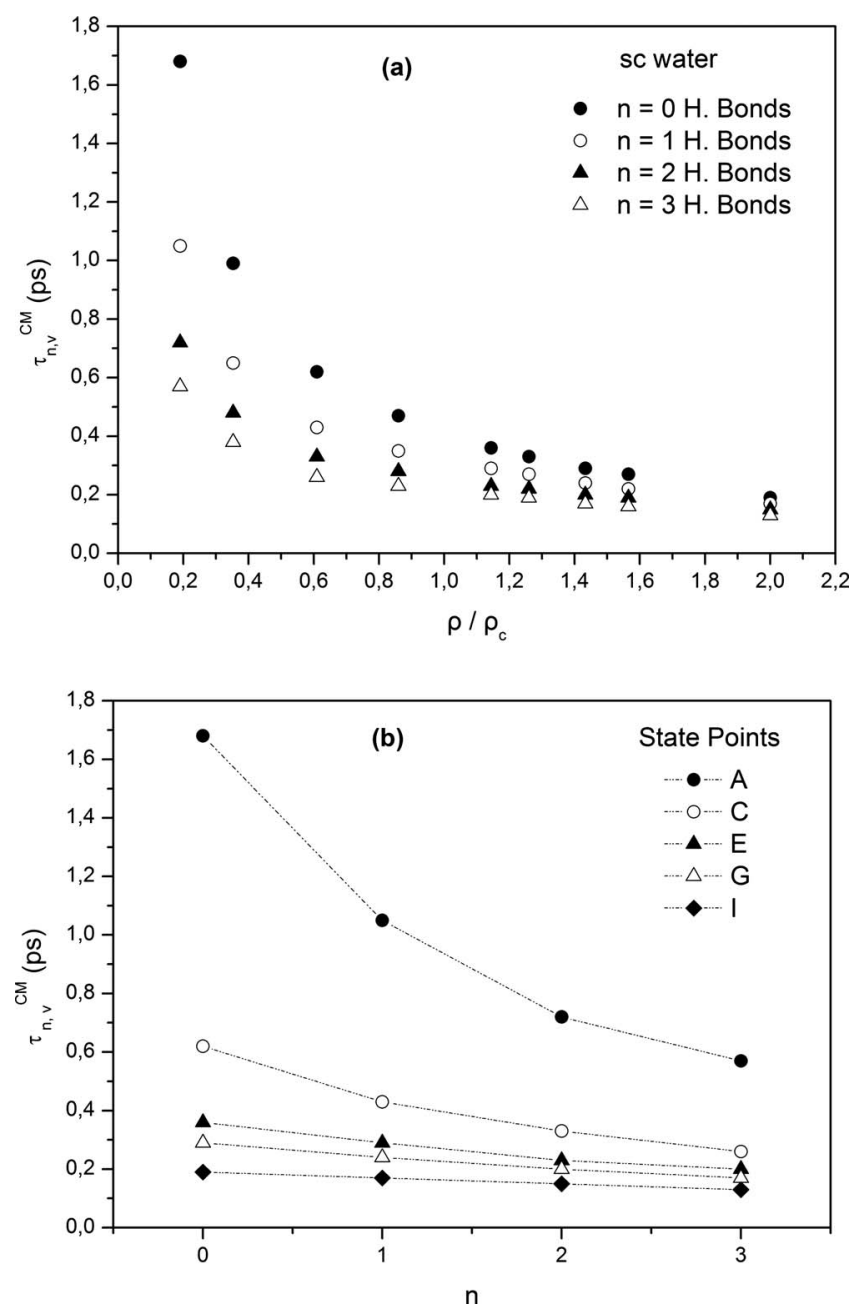

FIG. 8. CM velocity correlation time $\tau_{n, v}{ }^{\mathrm{CM}}$ as a function of (a) the bulk density of the system and (b) the number of $\mathrm{H}$. Bonds formed by a water molecule for several representative state points.

around each molecule and this frequency becomes higher for more strongly hydrogen bonded molecules.

However, the bulk density dependence of these libration frequencies, $\omega_{n, v, \max } \mathrm{H}$, is strongly affected by the number of hydrogen bonds $n$, as it may be clearly inspected in Fig. 10. From this figure we may observe that in the cases of weakly and hydrogen bonded free molecules $(n=0,1)$, the frequency $\omega_{n, v, \max }{ }^{H}$ increases almost linearly with density, whereas in the case of $n=2$ a clear deviation from linearity is observed. In the case of more strongly hydrogen bonded water molecules $(n=3)$, the frequency $\omega_{n, v, \max }^{\mathrm{H}}$ is not significantly affected by the bulk density of the system and exhibits a plateau located at about $406 \mathrm{~cm}^{-1}$. Therefore, we may conclude that the density dependence of the average libration peak frequencies $\omega_{v, \max }^{\mathrm{H}}$ at the range of investigated thermodynamic state points is mainly controlled by the change in the fraction of water molecules having zero, one, and two hydrogen bonds ${ }^{24}$ since for more strongly hydrogen bonded molecules, the density effects are almost negligible. Since the fraction of molecules having two hydrogen bonds increases significantly with the increase in the bulk density, ${ }^{24}$ whereas the fraction of free molecules decreases rapidly and the fraction of molecules having one hydrogen bond exhibit a maxi-
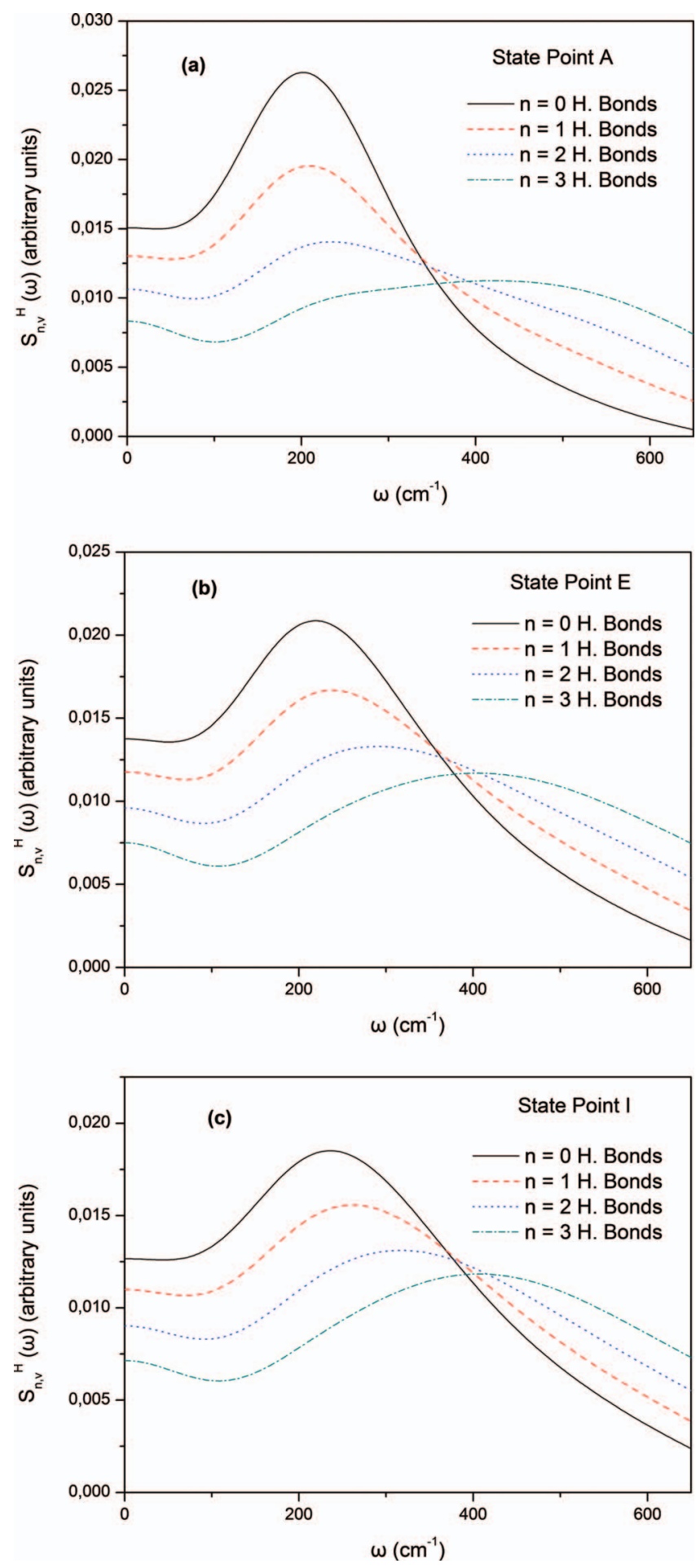

FIG. 9. The calculated spectral densities $S_{n, v}{ }^{\mathrm{H}}(\omega)$ [Eq. (11)] for some representative state points.

mum close to the critical density and then starts to decrease slightly, ${ }^{24}$ the contribution of molecules having two hydrogen bonds in the average libration spectrum starts to become more important as the density increases.

\section{CONCLUSIONS}

In the present treatment, MD simulation techniques have been employed in order to investigate systematically the effects of the local HB network on the single translational and 


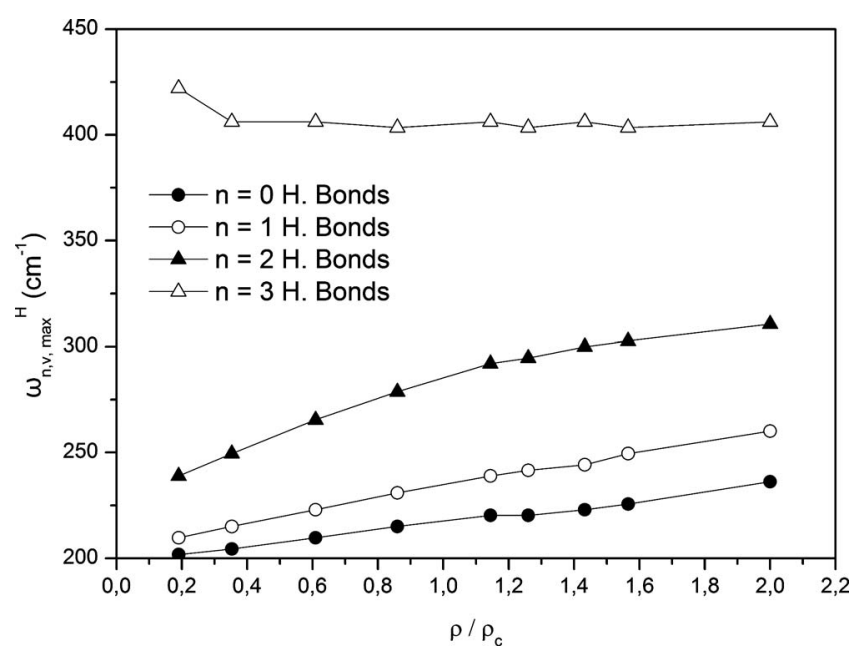

FIG. 10. Bulk density dependence of the calculated libration peak frequencies $\omega_{n, v, \max }{ }^{\mathrm{H}}$.

reorientational dynamics in SCW. From the results obtained we may clearly see that the number of hydrogen bonds, $n$, formed by each water molecule affects significantly its reorientational dynamic behavior. In general, we may observe a slower decay in the reorientational tcfs $C_{n, 1 R}(t)$ as the values of $n$ increase and this slowing down is reflected on the calculated reorientational correlation times $\tau_{n, 1-R}$. This behavior is more pronounced in the case of the first-order Legendre reorientational dynamics, whereas as in the case of the second-order Legendre reorientational dynamics and especially for HB-free molecules, the corresponding reorientational times are not significantly affected by the bulk density.

Moreover, we may clearly observe that the translational dynamics are also very sensitive on the HB state of the water molecules and the correlation times $\tau_{n, v}{ }^{\mathrm{CM}}$ exhibit, in general, a nonlinear decrease with density. Concerning the calculated spectral densities $S_{v}{ }^{\mathrm{H}}(\omega)$ of SCW, by calculating the corresponding libration peak frequencies $\omega_{v \text {,max }} \mathrm{H}$, we have revealed that their dependence on the effective local densities $\rho_{\text {eff }, l}$ of SCW is clearly nonlinear. This result could be regarded as an additional support of experimental observations suggesting the use of a nonlinear relation when analyzing the density dependence of spectroscopic peak frequencies in order to extract information about local density augmentation in supercritical fluids. Furthermore, these libration peak frequencies are shifted toward higher frequencies as the number of hydrogen bonds $n$ increases in the whole range of investigated densities. This blueshifting effect indicates clearly that the libration frequency of the water molecules is directly related to the local HB network around each molecule and this frequency becomes higher for more strongly hydrogen bonded molecules.

Finally concerning the HB dynamics in SCW, in general, we might say that although there is a strong interconnection between the static $\mathrm{HB}$ network and local density inhomogeneity effects in SCW, ${ }^{24}$ the HB dynamics seem to be not so strongly affected by the local structure of the system. Such an interrelation is observed only at very low bulk densities. At the low density region the calculated HB lifetimes are higher and this behavior has been related with the formation of small longer-lived clusters, which have been related in previous publications ${ }^{24,36,37}$ with the slowing down effects in the local density reorganization of the first solvation shell around the molecules in supercritical fluids.

\section{ACKNOWLEDGMENTS}

I.S. acknowledges the postdoctoral financial support of the Department of Physics and Nuclear Engineering (DFEN) (http://www.fen.upc.edu) of the Technical University of Catalonia (UPC), Barcelona-Spain and Dr. Dietmar Paschek (Center for Biotechnology and Interdisciplinary Studies, Rensselaer Polytechnic Institute, USA) for the fruitful discussion concerning specific technical details of the MOSCITO code. The CPU time allocation on the facilities of the Computer Simulation in Condensed Matter Research Group in DFEN is also gratefully acknowledged. E.G. gratefully acknowledges financial support from the Direcció General de Recerca de la Generalitat de Catalunya (Grant No. 2005SGR-00779) and from the Ministerio de Educación y Ciencia of Spain (Grant No. FIS2006-12436-CO2-01).

${ }^{1}$ H. Weingärtner and E. U. Franck, Angew. Chem., Int. Ed. 44, 2672 (2005).

${ }^{2}$ Supercritical Fluids: Fundamentals and Applications, NATO Advanced Studies Institute, Series E: Applied Science, edited by E. Kiran, P. G. Debenedetti, and C. J. Peters (Kluwer Academic, Dordrecht, 2000), Vol. 366.

${ }^{3}$ N. Akiya and Ph. Savage, Chem. Rev. (Washington, D.C.) 102, 2725 (2002).

${ }_{5}^{4}$ A. Kruse and E. Dinjus, J. Supercrit. Fluids 39, 362 (2007).

${ }^{5}$ P. Postorino, R. H. Tromp, M. A. Ricci, A. K. Soper, and G. W. Neilson, Nature (London) 366, 668 (1993).

${ }^{6}$ A. K. Soper, Chem. Phys. 258, 121 (2000).

${ }^{7}$ M. C. Bellissent-Funel, T. Tassaing, H. Zhao, D. Beysens, B. Guillot, and Y. Guissani, J. Chem. Phys. 107, 2942 (1997).

${ }^{8}$ Y. E. Gorbaty and A. G. Kalinichev, J. Phys. Chem. 99, 5336 (1995).

${ }^{9}$ M. M. Hoffmann and M. S. Conradi, J. Am. Chem. Soc. 119, 3811 (1997).

${ }^{10}$ A. Botti, F. Bruni, M. A. Ricci, and A. K. Soper, J. Chem. Phys. 109, 3180 (1998).

${ }^{11}$ A. K. Soper, F. Bruni, and M. A. Ricci, J. Chem. Phys. 106, 247 (1997).

${ }^{12}$ T. Tassaing, M. C. Bellissent-Funel, B. Guillot, and Y. Guissani, Europhys. Lett. 42, 265 (1998).

${ }^{13}$ R. H. Tromp, P. Postorino, G. W. Neilson, M. A. Ricci, and A. K. Soper, J. Chem. Phys. 101, 6210 (1994).

${ }^{14}$ N. Matubayasi, C. Wakai, and M. Nakahara, Phys. Rev. Lett. 78, 2573 (1997).

${ }^{15} \mathrm{Ph}$. Wernet, D. Testernale, J. L. Hazemann, R. Argoud, P. Glatzel, L. G. M. Pettersson, A. Nilsson, and U. Bergmann, J. Chem. Phys. 123, 154503 (2005).

${ }^{16}$ A. K. Soper, J. Phys.: Condens. Matter 8, 9263 (1996).

${ }^{17}$ P. Jedlovszky, J. B. Brodholt, F. Bruni, M. A. Ricci, A. K. Soper, and R. Vallauri, J. Chem. Phys. 108, 8528 (1998).

${ }^{18}$ A. G. Kalinichev and S. V. Churakov, Fluid Phase Equilib. 183, 271 (2001).

${ }^{19}$ A. G. Kalinichev and S. V. Churakov, Chem. Phys. Lett. 302, 411 (1999).

${ }^{20}$ T. I. Mizan, P. E. Savage, and R. M. Ziff, J. Phys. Chem. 100, 403 (1996).

${ }^{21}$ C. Nieto-Draghi, J. Bonet Avalos, and B. Rousseau, J. Chem. Phys. 118, 7954 (2003).

${ }^{22}$ J. Martí, Phys. Rev. E 61, 449 (2000).

${ }^{23}$ A. G. Kalinichev and J. D. Bass, J. Phys. Chem. 101, 9720 (1997).

${ }^{24}$ I. Skarmoutsos, D. Dellis, and J. Samios, J. Phys. Chem. B 113, 2783 (2009).

${ }^{25}$ G. Löfler, H. Schreiber, and O. Steinhauser, Ber. Bunsenges. Phys. Chem. 98, 1575 (1994).

${ }^{26}$ E. S. Fois, M. Sprik, and M. Parinello, Chem. Phys. Lett. 223, 411 
(1994).

${ }^{27}$ M. Boero, K. Terakura, T. Ikeshoji, C. C. Liew, and M. Parinello, J. Chem. Phys. 115, 2219 (2001).

${ }^{28}$ A. A. Chialvo and P. T. Cummings, J. Phys. Chem. 100, 1309 (1996).

${ }^{29}$ Y. Guissani and B. Guillot, J. Chem. Phys. 98, 8221 (1993).

${ }^{30}$ R. D. Mountain, J. Chem. Phys. 103, 3084 (1995).

${ }^{31}$ R. D. Mountain, J. Chem. Phys. 110, 2109 (1999).

${ }^{32}$ J. Martí, J. Chem. Phys. 110, 6876 (1999).

${ }^{33}$ I. Skarmoutsos and J. Samios, J. Phys. Chem. B 110, 21931 (2006).

${ }^{34}$ I. Skarmoutsos and J. Samios, J. Chem. Phys. 126, 044503 (2007).

${ }^{35}$ D. Dellis, I. Skarmoutsos, and J. Samios, "Molecular simulations of benzene and hexafluorobenzene using new optimized effective potential models: Investigation of the liquid, vapor-liquid coexistence and supercritical fluid phases," J. Mol. Liq. (in press).

${ }^{36}$ I. Skarmoutsos and E. Guardia, J. Phys. Chem. B 113, 8887 (2009).

${ }^{37}$ I. Skarmoutsos and E. Guardia, J. Phys. Chem. B 113, 8898 (2009).

${ }^{38}$ D. Paschek and A. Geiger, MOScITO 4.140, University of Dortmund, Dortmund, Germany, 2007.

${ }^{39}$ H. J. C. Berendsen, J. P. M. Postma, W. F. Van Gunsteren, A. Di Nola, and J. R. Haak, J. Chem. Phys. 81, 3684 (1984).

${ }^{40}$ J. P. Ryckaert, G. Ciccotti, and H. J. C. Berendsen, J. Comput. Phys. 23, 327 (1977).

${ }^{41}$ H. J. C. Berendsen, J. R. Grigera, and T. P. Straatsma, J. Phys. Chem. 91, 6269 (1987).

${ }^{42}$ E. Guàrdia and J. Marti, Phys. Rev. E 69, 011502 (2004).

${ }^{43}$ E. Johansson, K. Bolton, and P. Ahlström, J. Chem. Phys. 123, 024504 (2005).

${ }^{44}$ I. Brovchenko, A. Geiger, and A. Oleinikova, J. Chem. Phys. 123, 044515 (2005)

${ }^{45}$ I. Skarmoutsos, "Theoretical-computational investigation of the influence of thermodynamic parameters on the macroscopic, structural and dynamic properties of supercritical molecular systems," Ph.D. thesis, National and Kapodistrian University of Athens, 2006.

${ }^{46}$ D. C. Rapaport, Mol. Phys. 50, 1151 (1983).

${ }^{47}$ E. Guàrdia, D. Laria, and J. Marti, J. Phys. Chem. B 110, 6332 (2006)

${ }^{48}$ A. Luzar and D. Chandler, Nature (London) 379, 55 (1996).

${ }^{49}$ A. Chandra, Phys. Rev. Lett. 85, 768 (2000).

${ }^{50}$ M. Matsumoto and K. E. Gubbins, J. Chem. Phys. 93, 1981 (1990).

${ }^{51}$ E. Guardia, J. Marti, L. Garcia-Tarres, and D. Laria, J. Mol. Liq. 117, 63
(2005).

${ }^{52}$ B. S. Mallik and A. Chandra, J. Chem. Phys. 125, 234502 (2006).

${ }^{53}$ J. Martí, J. Padro, and E. Guardia, J. Chem. Phys. 105, 639 (1996).

${ }^{54}$ K. Yoshida, N. Matubayasi, and M. Nakahara, J. Chem. Phys. 127, 174509 (2007).

${ }^{55}$ E. Guàrdia and J. Marti, J. Mol. Liq. 101, 137 (2002).

${ }^{56}$ N. Matubayasi, N. Nakao, and M. Nakahara, J. Chem. Phys. 114, 4107 (2001).

${ }^{57}$ A. G. Kalinichev and K. Heinzinger, in Thermodynamic Data: Systematics and Estimation, Advances in Physical Geochemistry, edited by S. K. Saxena (Springer Verlag, New York, 1992), Vol. 10, pp. 1-59.

${ }^{58}$ A. G. Kalinichev, Ber. Bunsenges. Phys. Chem. 97, 872 (1993).

${ }^{59}$ B. D. Bursulaya and H. J. Kim, J. Chem. Phys. 110, 9656 (1999).

${ }^{60}$ S. C. Tucker, Chem. Rev. (Washington, D.C.) 99, 391 (1999)

${ }^{61}$ W. Song, R. Biswas, and M. Maroncelli, J. Phys. Chem. A 104, 6924 (2000).

${ }^{62}$ W. Song and M. Maroncelli, Chem. Phys. Lett. 378, 410 (2003).

${ }^{63}$ J. E. Lewis, R. Biswas, A. G. Robinson, and M. Maroncelli, J. Phys. Chem. B 105, 3306 (2001)

${ }^{64}$ M. Besnard, T. Tassaing, Y. Danten, J. M. Andanson, J. C. Soetens, F. Cansell, A. Loppinet-Serani, H. Reveron, and C. Aymonier, J. Mol. Liq. 125, 88 (2006).

${ }^{65}$ M. I. Cabaço, S. Longelin, Y. Danten, and M. Besnard, J. Phys. Chem. A 111, 12966 (2007).

${ }^{66}$ K. Saitow, K. Otake, H. Nakayama, K. Ishii, and K. Nishikawa, Chem. Phys. Lett. 368, 209 (2003).

${ }^{67}$ K. Saitow, H. Ohtake, N. Sarukura, and K. Nishikawa, Chem. Phys. Lett. 341, 86 (2001)

${ }^{68}$ K. Saitow, H. Nakayama, K. Ishii, and K. Nishikawa, J. Phys. Chem. A 108, 5770 (2004)

${ }^{69}$ K. Saitow and J. Sasaki, J. Chem. Phys. 122, 104502 (2005).

${ }^{70}$ P. Lalanne, T. Tassaing, Y. Danten, F. Cansell, S. C. Tucker, and M. Besnard, J. Phys. Chem. A 108, 2617 (2004).

${ }^{71}$ K. Yui, H. Uchida, K. Itatani, and S. Koda, Chem. Phys. Lett. 477, 85 (2009).

${ }^{72}$ S. A. Egorov, J. Chem. Phys. 113, 1950 (2000).

${ }^{73}$ Y. Yasaka, M. Kubo, N. Matubayasi, and M. Nakahara, Bull. Chem. Soc. Jpn. 80, 1764 (2007) 